\title{
Mecanismos del ciclo celular y la apoptosis implicados en las resistencias a los fármacos de uso intravesical en el cáncer superficial de vejiga
}

\author{
J.P. Burgués Gasión, J.L. Pontones Moreno*, C.D. Vera Donoso*, J.F. Jiménez Cruz*, \\ M. Ozonas Moragues \\ Servicio de Urología. Hospital Universitario Son Dureta Palma de Mallorca. *Servicio de Urología. \\ Hospital Universitario La Fe. Valencia. \\ Actas Urol Esp 2005; 29 (9): 846-859
}

\section{RESUME N}

MECANISMOS DEL CICLO CELULAR Y LA APOPTOSIS IMPLICADOS EN LAS RESISTENCIAS A LOS FÁRMACOS DE USO INTRAVESIAL EN EL CÁNCER SUPERFICIAL DE VEJIGA

Numerosos estudios han demostrado la utilidad de la quimioterapia intravesical en la profilaxis de las recidivas del tumor vesical superficial después de la resección transuretral. Pero la eficacia de esta práctica puede verse limitada por la existencia de resistencias a agentes individuales o de multirresistencias a varios fármacos. Además de los mecanismos de resistencia clásicos o "unicelulares" determinados genéticamente, como la expresión de la glicoproteína P-170 (gen $m d r$-1), la sobreexpresión del gen Bcl-2 o la actividad de la glutation S-transferasa, existen otros mecanismos de resistencia "multicelular" que han podido ser demostrados en cultivos de agregados celulares tridimensionales, y no en las mismas células cultivadas en monocapa o en suspensión de células individuales. La adhesión célula-célula y célula-estroma condiciona por un lado una limitada penetración de las drogas y, por otro, unos gradientes de oxígeno y proliferación en los tejidos que crean distintas susceptibilidades al efecto citotóxico. El conocimiento de las resistencias y sus mecanismos es de gran importancia, puesto que se trata de uno de los puntos donde se puede actuar para optimizar la eficacia de la quimioterapia intravesical. Muchos investigadores han dedicado sus esfuerzos a la búsqueda de medios contra las resistencias mediante la aplicación de métodos para mejorar la penetración de los fármacos, la búsqueda de agentes reversores o la identificación de las resistencias con tests in vitro de quimiosensibilidad.

Palabras clave: Quimioterapia intravesical. Quimiorresistencia. Tumor vesical superficial.

\begin{abstract}
CELL CYCLE AND APOPTOSIS MECHANISMS IMPLICATED IN INTRAVESICAL CHEMOTHERAPY RESISTANCES IN SUPERFICIAL BLADDER CANCER

It is well documented the effectiveness of intravesical chemotherapy following transurethral resection to prevent recurrences of superficial bladder cancer. But it is also known that efficacy may be limited by tumour cell resistance to one or several of the drugs available for instillation. In addition to the genetically determined unicellular mechanisms classically described in the literature such as glycoprotein P-170 expression (mdr-1), overexpression of Bcl-2 or glutation S-transferase activity, it has been recently shown that multicellular mechanisms may also be involved in drug resistance. Multicellular resistance can only be demonstrated in three-dimensional cultures and fails to be shown in monolayers or cell suspensions. This is explained by the fact that cell-to-cell and cell-to-stroma adhesion limits drug penetration and by the variable susceptibility to cytotoxicity determined by oxygen and tissue proliferation gradients. A better understanding of the molecular mechanisms involved in drug resistance is necessary to increase intravesical chemotherapy effectiveness. Current research includes improving drug penetration, searching resistance reversing agents and developing in vitro chemosensitivity tests to identify drug resistance.
\end{abstract}

Keywords: Intravesical chemotherapy. Drug resistance. Superficial bladder neoplasms. 
$\mathrm{N}$ umerosos estudios han mostrado la eficacia de la quimioterapia intravesical en la profilaxis de las recidivas de los tumores vesicales superficiales después de la resección transuretral (RTU) ${ }^{1-4}$. En los tumores con alto riesgo de progresión como el T1G3 o el CIS se prefiere como tratamiento adyuvante de primera línea el uso de la inmunoterapia con bacilo de CalmetteGuérin (BCG), pero en el caso de tumores de riesgo bajo o intermedio como los Ta, los T1G1 y los T1G2 la Asociación Europea de Urología, en sus Guidelines on non-muscle invasive bladder cancer $^{5}$ aconsejan el uso de agentes quimioterápicos, dado que los efectos secundarios tanto locales como sistémicos son significativamente inferiores.

La reducción en las tasas de recurrencia a un año de los tumores vesicales superficiales con el uso de la quimioterapia intravesical alcanza el $44 \%$, si lo comparamos con aquellos pacientes tratados sólo con RTU6 ${ }^{6}$. Estudios más a largo plazo sugieren sin embargo, que cuando se sigue a los pacientes durante un periodo largo de tiempo las tasas de recidiva tienden a aumentar y las diferencias entre la RTU aislada y seguida de quimioprofilaxis se hacen mucho menores ${ }^{7}$. Aunque la eficacia a largo plazo queda pues cuestionada, se acepta una reducción de un 20-30\% en la tasa global de recidiva, una disminución al 50\% en el número de recidivas anuales, y un aumento significativo del intervalo libre de enfermedad hasta la aparición de la primera recidiva ${ }^{8}$. Además de la terapia profiláctica de las recidivas, la quimioterapia intravesical se ha mostrado también útil como terapia ablativa de lesiones residuales o no asequibles a la cirugía, de pequeñas recidivas incipientes, o bien de tumores primarios en pacientes con contraindicación absoluta a ser intervenidos ${ }^{9}$.

Existen varios agentes farmacológicos de uso común en nuestro medio para la prevención de recidivas de tumor vesical superficial como la tiotepa (TIO), mitomicina C (MMC), adriamicina (ADR) y epirubicina (EPI). Todos han resultado ser eficaces en los distintos estudios cuando se han utilizado intravesicalmente en instilaciones repetidas $^{1,3,4}$. Según un metaanálisis de Kamat et $\mathrm{al}^{2}$ hay un $44 \%$ de recurrencias con el uso de TIO intravesical, un $39 \%$ con $\mathrm{ADR}$, un $36 \%$ con MMC y un $39 \%$ con EPI. Por tanto, a pesar de la profilaxis con quimioterapia intravesical sigue existiendo un elevado índice de recurrencias.

Se sabe que pueden existir resistencias que condicionen el fracaso de la quimioprofilaxis en los tumores vesicales superficiales. Estas resistencias pueden ser a fármacos individuales o a varios fármacos (multirresistencias), son variables de un tumor a otro y se desconocen cuando se trata al paciente. La elección del agente en la práctica clínica se realiza de modo empírico, sólo en base a la experiencia o disponibilidad que se tiene con alguno de ellos.

Además de los mecanismos de resistencia "clásicos" o "unicelulares" determinados genéticamente, como la expresión de la glicoproteína P170 (gen $m d r-1$ ) o de las proteínas relacionadas con la multirresistencia (MRP), la sobreexpresión del gen $\mathrm{Bcl}-2$ o los niveles intracelulares de glutation (GSH) y la actividad de la glutation S-transferasa (GST), existen otros mecanismos de resistencia "multicelular" que han podido ser demostrados en cultivos de agregados celulares tridimensionales, y no en las mismas células cultivadas en monocapa o en una suspensión de células individuales. La adhesión célula-célula y célulaestroma condiciona por un lado una limitada penetración de las drogas y, por otro, unos gradientes de oxígeno y proliferación en los tejidos que crean distintas susceptibilidades al efecto citotóxico.

El conocimiento de las resistencias y sus mecanismos es de gran importancia, puesto que se trata de uno de los puntos donde se puede actuar para optimizar la eficacia de la quimioterapia intravesical. Muchos investigadores han dedicado sus esfuerzos a la búsqueda de medios contra las resistencias, unas veces con métodos para mejorar la penetración de las drogas en la pared vesical, otras con agentes que las reviertan y otras identificándolas mediante tests in vitro de quimiosensibilidad.

En la presente revisión, se comenzará con un breve resumen de los procesos biológicos que acontecen durante el ciclo celular y la apoptosis, cuyo conocimiento es esencial para entender los mecanismos de las resistencias. A continuación se expondrán los mecanismos unicelulares y multicelulares que se han identificado como cau- 
santes de las resistencias en los tumores vesicales. Finalmente, se mostrarán algunos ejemplos que ilustran cómo el conocimiento de las resistencias puede tener su aplicación en la práctica clínica.

\section{CICLO CELULAR Y APOPTOSIS}

Para entender las bases moleculares del cáncer, el mecanismo de acción de los agentes quimioterápicos y los mecanismos de la quimiorresistencia es necesario conocer los procesos biológicos que regulan el ciclo celular y la apoptosis.

En condiciones normales existe un equilibrio entre la proliferación celular y la apoptosis (Fig. 1), pero en el cáncer aparecen mutaciones que dan lugar a la activación de oncogenes (codifican proteínas del ciclo celular que favorecen la proliferación celular) o a la inactivación de genes supresores (codifican proteínas que frenan el ciclo celular). De este modo el equilibrio se rompe y la balanza se decanta hacia la proliferación desenfrenada y la inmortalización de la línea celular. Pero un cáncer se define no sólo por la inmortalización de las células sino también por la capacidad invasora local y a distancia, para lo cual son necesarias mutaciones adicionales. Aquellas mutaciones que favorezcan la angiogénesis mediada por los factores de crecimiento

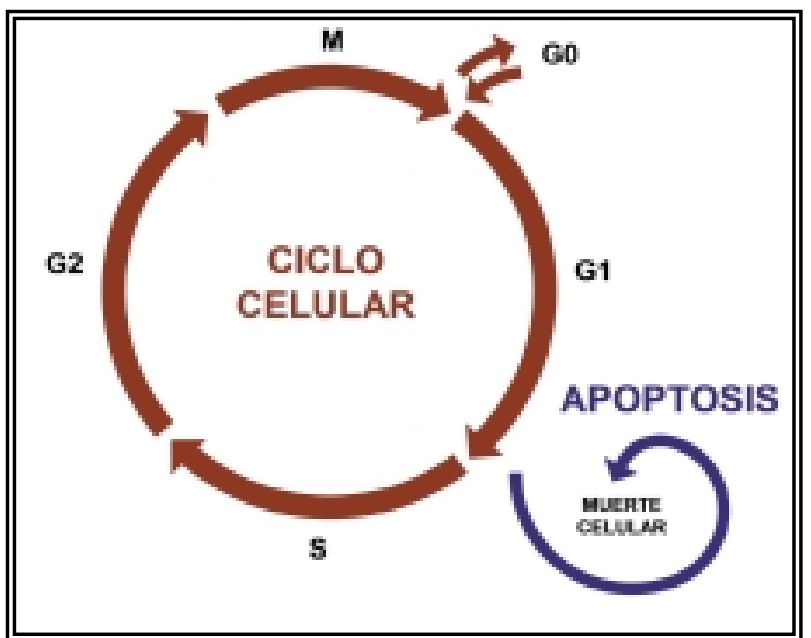

FIGURA 1: Ciclo celular y apoptosis. En el ciclo celular se distinguen varias fases: GO. Fase de reposo; G1. Fase de presintesis; S. Fase de sintesis; G2. Fase de postsintesis; $M$. Mitosis. En la apoptosis se produce la activación de las caspasas que inducen la condensación de la cromatina y la fragmentación del ADN, la destrucción nuclear y citoplásmica y, por último, la fagocitosis por macrófagos.
VEGF y PD-EDGF y la adhesión intercelular o a membranas basales mediada por las e-cadherinas, integrinas, cateninas, tailinas y otras moléculas de adhesión (genes cary nm-23) serán decisivas en la agresividad local y la capacidad metastásica del tumor.

\section{CICLO CELULAR}

El ciclo celular regula la división celular y es fundamental tanto para generar nuevas células en el desarrollo embrionario como para reemplazar las células dañadas en un órgano adulto. Se distinguen varias fases (Fig. 1): en la fase $G_{0}$ o de reposo las células permanecen sin dividirse, pero conservan la capacidad de reiniciar el ciclo para repoblar un tejido; en la fase $\mathrm{G}_{1}$ (gap 1) de presíntesis, las células producen ARN y proteínas; en la fase $\mathrm{S}$ se replica el $\mathrm{ADN}$; en la fase $\mathrm{G}_{2}$ (gap 2) o de postsíntesis el núcleo se organiza para la división celular, que se produce en la fase $\mathrm{M}$ o de mitosis. El ciclo completo dura desde 12 horas hasta meses o años. Mientras la mitosis tiene una duración constante de 1 hora, las células pueden permanecer "arrestadas" en $\mathrm{G}_{0}$ durante años.

Las moléculas reguladoras del ciclo celular son universales y están muy conservadas durante la evolución. Distintas proteínas (Fig. 2) ejercen el control en la entrada y la progresión de las células a lo largo de las fases del ciclo ${ }^{10-11}$ :

- Protein-kinasas dependientes de ciclinas (cyclin-dependent kinases o CDKs): fosforilan elementos claves en la progresión del ciclo celular. Para ser activas requieren unirse a las ciclinas. Hay tres tipos: CDKs $\mathrm{G}_{1}$ (CDKs 4, 6 y 2), CDKs fase $\mathrm{S}$ (CDK2) y CDKs mitóticas (CDKs 2 y 1).

- Ciclinas: proteínas que se unen a las CDKs y controlan su capacidad de fosforilación. Se denominan ciclinas porque se sintetizan y degradan durante el ciclo celular. Hay al menos 6 tipos de ciclinas distintas en mamíferos: A, B, C, D, E, F. También se clasifican en: ciclinas $G_{1}\left(D_{1}, D_{2}, D_{3}\right.$ y E), ciclinas fase $S$ (A y E) y ciclinas mitóticas (A y B). Las ciclinas $D_{1}, D_{2}$ y $D_{3}$ se sobreexpresan gracias a estímulos mitogénicos como factores de crecimiento y se unen a las CDKs 4 y 6 que fosforilan parcialmente a la proteína del retinoblastoma (pRb) haciendo que la célula progrese en la fase $G_{1}$ temprana y no quede arrestada en $G_{0}$. 


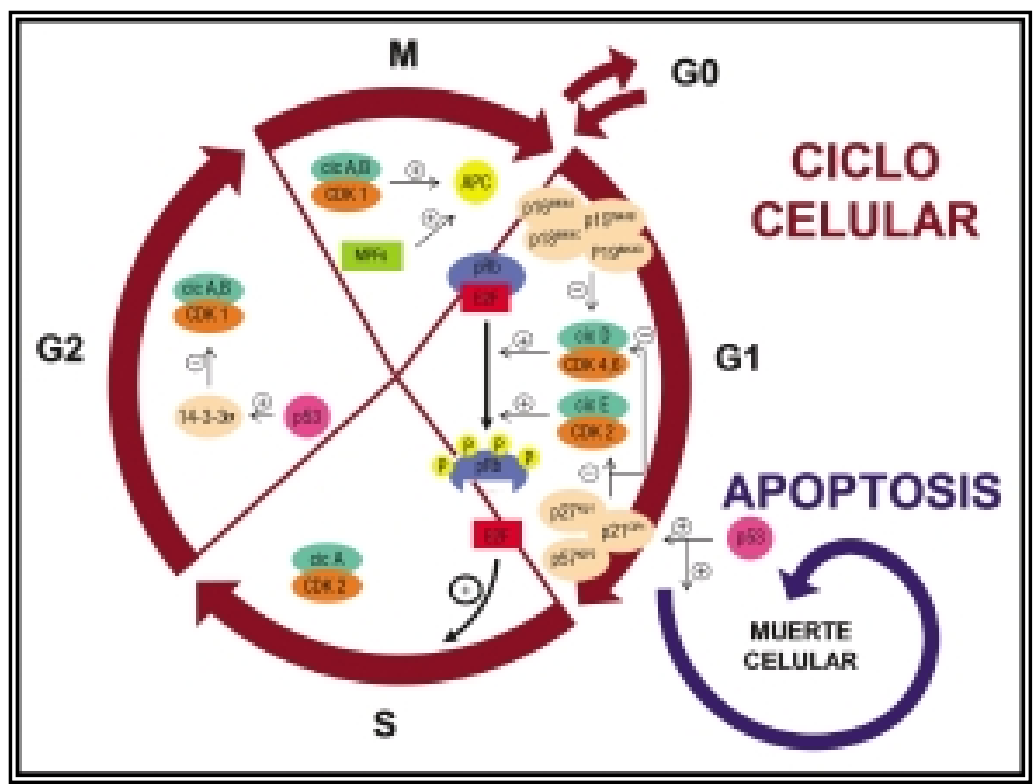

FIGURA 2: Moléculas reguladoras del ciclo celular. Cic: ciclinas. pRb: proteina del retinoblastoma. CDK: kinasa ciclina-dependiente. E2F: factor de transcripción. MPF: factor de maduración-promoción. APC: complejo promotor de la anafase.

Los complejos ciclina E - CDK2 se activan en la fase $\mathrm{G}_{1}$ tardía completando la fosforilación de la pRb.

- Inhibidores de las CDK (cyclin-dependent kinases inhibitors o CDKIs): existen dos familias de CDKIs, la familia del inhibidor de la CDK4 (INK4) y la familia de la proteína inhibidora de CDK (CIP / KIP). Las proteínas de la familia INK4 (INK4A, INK4B, INK4C e INK4D) inhiben específicamente a la CDK4 y la CDK6 en $\mathrm{G}_{1}$, mientras que los inhibidores de la familia CIP / KIP (CIP1, KIP1 y KIP2) inhiben a las CDKs en todas las fases del ciclo. Las CDKIs mejor conocidas son la p16 ${ }^{\text {INK4A }}$ (gen $m t s-1$ ), la p15 $5^{\text {INK4B }}$ (gen $m t s-2$ ), la $\mathrm{p} 18^{\mathrm{INK} 4 \mathrm{C}}$, la p19 ${ }^{\mathrm{INK} 4 \mathrm{D}}$, la $21^{\mathrm{CIP} 1}$ (gen waf-1) y la p27 ${ }^{\mathrm{KIP} 1}$ (gen kip-1).

- Proteína del retinoblastoma ( $p R b)$ y factor de transcripción E2F: la pRb forma un complejo con miembros de la familia del factor de transcripción $\mathrm{E} 2 \mathrm{~F}$. Cuando la pRb es fosforilada por las CDKs se libera el E2F, que induce el paso de $\mathrm{G}_{1}$ a $\mathrm{S}$ al activar la maquinaria de sintesis del ADN. Recientemente se ha descrito el E2F6, una forma truncada e inactiva del E2F que se une a la pRb en las células en $\mathrm{G}_{0}$.

- Factor de maduración-promoción (maturationpromoting factor o MPF): protein-kinasa que en la meiosis induce la maduración de los oocitos, y en la mitosis interviene en la transición de $G_{2}$ a $M$. Una vez activado por las ciclinas, fosforila proteínas implicadas en la rotura de la membrana nuclear, la condensación de los cromosomas y la degradación de las ciclinas.

- Complejo promotor de la anafase (anaphase-promoting complex o APC): recientemente descubierto, es capaz de iniciar la separación de las cromátides hermanas en la anafase, favoreciendo la progresión de la fase $\mathrm{M}$.

- Ubiquitinas y proteosomas: las ubiquitinas son pequeñas proteínas que por acción de las enzimas activadora E1, conjugadora de ubiquitinas E2 y ubiquitín ligasa E3 se adhieren a un sustrato haciéndolo susceptible de la proteolisis por un proteosoma. Los proteosomas son complejos proteicos formados por múltiples subunidades responsables de la degradación de las ciclinas y CKIs una vez han efectuado su acción fosforiladora en cada fase. Una vez degradadas se hace irreversible la marcha atrás del ciclo.

- Proteína p53 (gen supresor TP53): activa a la p21 $1^{\mathrm{CIP} 1}$, CDKI que inhibe la fosforilación de la pRb impidiendo la liberación del E2F, deteniendo el ciclo en $\mathrm{G}_{1}$ e induciendo apoptosis. También puede detener el ciclo en $G_{2}$ mediante la activación de la proteína $14-3-3 \sigma$, que secuestra el complejo ciclina B - CDK 1 fuera del núcleo. Además, la p53 activa genes reparadores del ADN (mlh-1 y mlh-2) protegiendo de mutaciones y confiriendo estabilidad genética. El gen $M D M-2$ contrarregula a la p53 ubiquitinándola para hacerla susceptible a la degradación proteosómica.

- Otras proteínas: factores de crecimiento (todos son promitóticos excepto el TGF-b1), proteínas promitóticas de la familia Bcl-2 (Bcl-2, Bcl$\mathrm{xL}$ ), proteínas proapoptóticas de la familia $\mathrm{Bcl}-2$ (Bax, Bim, Bid, Bad y Bak).

Las células tienen mecanismos de control capaces de frenar el ciclo celular si algún error ocurre, bien para poder ser reparado, o bien para inducir la apoptosis cuando el daño es muy extenso. En condiciones normales no se permite la progresión del ciclo hasta que el error no se 
haya corregido; sin embargo, las células tumorales tienen mutada alguna de las proteínas reguladoras y son capaces de repetir el ciclo indefinidamente aún sin integridad del ADN.

En cualquier tejido y también en los tumores existe un equilibrio entre las células que se encuentran en fase $G_{1}$ con las que están en reposo en $G_{0}$. Las células en $G_{0}$ son relativamente inactivas con respecto a la síntesis de macromoléculas y, en consecuencia, insensibles a muchos de los antineoplásicos, particularmente a los que afectan a esta síntesis. Los agentes quimioterápicos que actúan sobre las células en división más que sobre las células en $\mathrm{G}_{0}$ se denominan "específicos del ciclo celular", mientras que aquellos que actúan también sobre las células en $\mathrm{G}_{0}$ reciben el nombre de "no específicos del ciclo celular". A su vez, los específicos del ciclo celular pueden ser "fase específicos" si actúan sólo sobre alguna de las fases, o "no fase específicos"12. Los agentes más frecuentemente utilizados en instilación endovesical (tiotepa, adriamicina, epirubicina y mitomicina C) son todos más activos en las células en división, pero no se consideran específicos del ciclo celular porque en alguna medida también actúan sobre las células en $\mathrm{G}_{0}$.

\section{APOPTOSIS}

La apoptosis es la forma fisiológica de suicidio celular que interviene en la embriogénesis, en el recambio celular de los tejidos y en la eliminación de células infectadas, mutadas o dañadas. Morfológicamente, la diferencia con la necrosis es que en la apoptosis se produce la fragmentación de ADN mientras se mantiene la integridad de la membrana hasta estadios tardíos en la muerte celular. Bioquímicamente, la característica diferencial es que se trata de un proceso dependiente de energía y de la síntesis de nuevas proteínas, mientras que en la necrosis falla el aporte de energía y la síntesis de proteínas se interrumpe ${ }^{13}$. En el proceso de apoptosis se distinguen varias fases: en la fase $D_{1}$ se producen los mecanismos moleculares que inician el fenómeno; en la fase $\mathrm{F}$ se fragmenta el $\mathrm{ADN}$; en la fase $\mathrm{D}_{2}$ se produce la destrucción nuclear y citoplásmica, y los restos son fagocitados por macrófagos ${ }^{14}$.

Los procesos morfológicos y bioquímicos que caracterizan a la apoptosis están mediados por "efectores de muerte celular" como las caspasas (proteasas cisteínicas). Pero para que estos efectores actúen es necesario que el balance vidamuerte se rompa por la interacción de los "activadores de muerte celular", extracelulares o intracelulares ${ }^{13}$ :

- Activadores de muerte extracelulares: la supervivencia celular depende de la recepción de señales extracelulares adecuadas, en ausencia de las cuales la célula se suicida por el mecanismo de la apoptosis. Algunos ejemplos son:

- Integrinas: son moléculas que conectan la matriz extracelular con el citoesqueleto celular. Pero además de su función estructural actúan como transductores de señales de supervivencia celular y de protección frente a la apoptosis cuando el contacto con la matriz extracelular es adecuado.

- Factores de crecimiento: la supervivencia celular también depende de una exposición apropiada a factores de crecimiento.

- Citokinas de muerte celular: son capaces de iniciar directamente la muerte vía apoptosis. Es el caso del ligando Fas y del factor de necrosis tumoral (tumoral necrosis factor o TNF), producidos por los linfocitos $\mathrm{T}$ y las células natural killer.

- Activadores de muerte intracelulares: un ejemplo es la familia de proteínas Bcl-2, involucradas en el control de la apoptosis. Algunos miembros de la familia son anti-apoptóticos (Bcl2 y Bcl-xL) y otros son pro-apoptóticos (Bax, Bim, Bid, Bad y Bak).

Las señales apoptóticas, tanto intra como extracelulares, convergen para activar a las caspasas. La condensación de la cromatina, la fragmentación del ADN, la rotura de la membrana nuclear y la formación de cuerpos apoptóticos son consecuencias directas de la actividad caspasa. Las caspasas en las células vivas existen como zimógenos inactivos que se activan por rotura proteolítica mediante dos vías alternativas que se ilustran en la Figura 3 y se describen a continuación ${ }^{13,15}$.

- Vía del receptor de muerte celular: los receptores de muerte pertenecen a la superfamilia génica de receptores del TNF (TNFR). Los mejor caracterizados son el receptor Fas, también conocido como CD95 (cytotoxicity-dependent protein 95) o APO-1 (apoptosis-1 protein), y el receptor del 


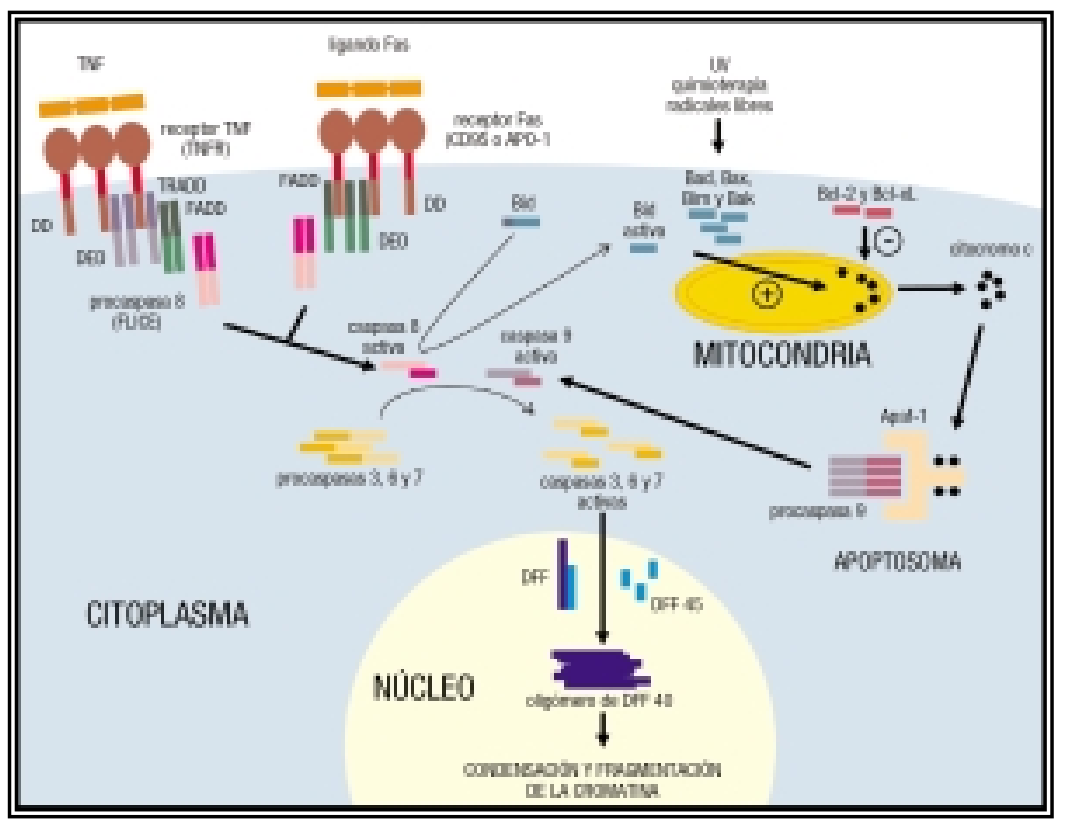

FIGURA 3: Vías apoptóticas celulares: vía del receptor de muerte (TNF o ligando Fas/FADD/caspasa 8) y via mitocondrial (familia Bcl-2/citocromo c/ Apaf-1/caspasa 9).

TNF (TNFR). Tienen en común una secuencia homóloga denominada "dominio de muerte" (death domain o DD), capaz de iniciar la cascada de eventos que llevan a la apoptosis. Los ligandos para estos receptores pertenecen a la familia del TNF, y son el ligando Fas y el TNF. Estos ligandos, que normalmente existen en forma trimérica, se unen a sus receptores induciendo también su trimerización y así activándolos. El receptor Fas activado recluta moléculas adaptadoras como el FADD / MORT-1 (Fas-associated death domain / mediator of receptor-induced toxicity-1), que contiene también un "dominio de muerte" (DD) que se une al análogo del receptor Fas y un "dominio efector de muerte" (DED) que se une a un dominio análogo de la procaspasa 8. Esta enzima, también conocida como FLICE (FADDlike IL-1ß-converting enzyme), sufre activación autocatalitica al unirse al FADD convirtiéndose en la caspasa 8 activa.

$\mathrm{El}$ complejo que forman el Fas, el FADD y la FLICE se denomina DISC (death-inducing signaling complex). La caspasa 8 rompe y activa otras caspasas efectoras como la 3, la 6 y la 7 , que constituyen la principal actividad caspasa en las células apoptóticas. En el caso de los receptores del TNF (TNFR-I y el TNFR-II), la molécula adap- tadora que reclutan es el TRADD (TNFR-associated death domain), que a su vez sirve de plataforma para el reclutamiento de otros adaptadores como el FADD, el TRAF-2 (TNFRassociated factor-2) y el RIP (receptor interactive protein). El complejo formado finalmente activa las caspasas reguladoras y efectoras, al igual que lo hacía el receptor Fas ${ }^{16}$.

- Vía mitocondrial: se trata de una vía independiente de los receptores de muerte y es la que inducen los agentes quimioterápicos, la radiación ultravioleta o las moléculas de estrés (especies reactivas del oxígeno y del nitrógeno). Las mitocondrias son orgánulos con dos compartimentos: la matriz, rodeada por la membrana interna (MI), y el espacio intermembranal, entre las membranas interna y externa (ME). En la MI está la ATP sintetasa y la cadena respiratoria y en el espacio intermembrana está el citocromo c, que si se libera al exterior de la mitocondria induce la apoptosis. Las proteínas de la familia Bcl-2 regulan esta liberación: los miembros anti-apoptóticos de esta familia (Bcl-2 y Bcl$\mathrm{xL}$ ) bloquean la liberación del citocromo $\mathrm{c}$, mientras que los miembros pro-apoptóticos (Bax, Bim, Bid, Bad y Bak) la inducen. Cuando el citocromo c se libera se provoca el ensamblamiento del apaf-1 (apoptotic protease-activating factor) con la procaspasa 9, formándose el complejo denominado apoptosoma. El apaf-1 tiene un dominio reclutador de caspasas denominado CARD (caspase recruiting domain), que requiere ATP para la inducción de la ruptura autolítica de la procaspasa 9 en caspasa 9 activa. Esta última se libera del apoptosoma para activar a las caspasas 3,6 y 7 , que provocan la ruptura de diversos sustratos y la apoptosis.

Las señales de supervivencia extracelulares inhiben la apoptosis por activación de una kinasa que fosforila a la proteína Bad haciendo que esta quede secuestrada en el citoplasma en lugar de ejercer su acción pro-apoptótica en la mitocondria. Por el contrario, las señales apoptóticas extracelulares traslocan a Bad y Bax al interior de la mitocondria, provocando así la liberación del citocromo c en induciendo la activación de las 
caspasas. La proteína Bid puede ser traslocada al interior de la mitocondria por la caspasa 8 (activada por el ligando Fas o el TNF), amplificándose así la apoptosis iniciada por la vía del receptor de muerte.

\section{MECANISMOS DE RESISTENCIA}

Una vez vistos los procesos biológicos que regulan el ciclo celular y la apoptosis, se expondrán los mecanismos unicelulares y multicelulares que se han descrito en la literatura como causantes de las resistencias a los fármacos de uso intravesical.

\section{RESISTENCIA UNICELULAR}

Los mecanismos de resistencia "unicelular" están determinados genéticamente. Las resistencias pueden ser inherentes a la propia estirpe tumoral, pero también pueden aparecer de novo durante la evolución de la neoplasia por las mutaciones que se van produciendo en las células debido a su inestabilidad genética. La aplicación de drogas puede inducir una selección natural por el sobrecrecimiento de aquellos clones resistentes.

Se sabe que pueden existir resistencias individuales a un solo agente o bien multirresistencias a varios fármacos. En el caso de los tumores vesicales se han podido demostrar distintos mecanismos de resistencia celular a los quimioterápicos, todos ellos relacionados con la expresión de algún gen. Estos genes codifican proteínas que actúan unas veces como un canal que transporta al fármaco fuera de su lugar de acción (glicoproteína-P y proteínas relacionadas con la multirresistencia), otras inhibiendo la apoptosis (Bcl-2 y Bcl-xL), otras como una enzima detoxificadora (glutation-S-transferasa), en otras ocasiones la proteína se une al fármaco impidiendo su acción (metalotioneína), otras veces interviene en los procesos de replicación y reparación del ADN (topoisomerasa II- $\alpha$ ), otras bloquea el receptor Fas impidiendo que los linfocitos T lleven a cabo la apoptosis receptor-inducida (proteína FLICE inhibitoria), y otras veces se sabe sólo su relación con la quimiorresistencia pero no se conoce el mecanismo.

- Sobreexpresión de la glicoproteína P-170 (gen multidrug resistance-1 o mdr-1): Se trata de una proteína situada en la membrana nuclear y citoplásmica que actúa como un canal de flujo ATPdependiente que bombea los fármacos al exterior. Cuando la aplicación prolongada a un fármaco induce la sobreexpresión de la glicoproteína-P, frecuentemente aparecen resistencias cruzadas con otros fármacos no relacionados, convirtiendo a la célula en multirresistente. En vejigas normales pediátricas y de adultos no se expresa, en los tumores transicionales no tratados lo hace débilmente, y en los tratados con quimioterapia intravesical o sistémica la expresión está aumenta$\mathrm{da}^{17}$. La exposición continuada a ADR en una línea celular sensible provocó su transformación en otra resistente, con sobreexpresión de la glicoproteína-P detectable por inmunohistoquimia, y acúmulo del fármaco en las células demostrable con el microscopio de fluorescencia ${ }^{18}$. Estudios similares con $\mathrm{MMC}^{19}$ o con cisplatino ${ }^{20}$ también han demostrado la aparición de resistencias mediadas por la sobreexpresión del mdr-1. Algunos estudios confieren valor pronóstico a la expresión de la glicoproteína $\mathrm{P}-170^{21-23}$, pero otros no han sido capaces de demostrarlo ${ }^{24}$.

- Sobreexpresión de las proteínas relacionadas con la multirresistencia (multidrug resistanceassociated proteins, MDR associated proteins o $M R P)$ : Son un grupo de proteínas transportadoras ATP-dependientes que se sitúan en la membrana celular. Se han identificado 6 subfamilias dentro de las MRP humanas. En un estudio en una línea celular la sobreexpresión de las MRP fue inducida por el cisplatino a medida que las células iban adquiriendo resistencia ${ }^{20}$. En otro estudio similar una línea celular de tumor vesical fue expuesta a dosis crecientes de ADR hasta que se hizo resistente. Se observó la sobreexpresión de las MRP como mecanismo de la resistencia ${ }^{25}$. La expresión de MRP-1, MRP-2 y MRP-3 en tumores recurrentes y en tumores residuales tras tratamiento con ADR fue mucho mayor que en tumores primarios no tratados ${ }^{26}$.

- Sobreexpresión de la proteína pulmonar relacionada con la resistencia (lung resistance-related protein, LRP): La LRP se sobreexpresa en $2 / 3$ de los tumores vesicales primarios nunca tratados, $\mathrm{y}$ lo hace en mayor medida en bajos grados y estadios. Este hecho sugiere que la LRP puede constituir un mecanismo de multirresistencia 
intrínseco del cáncer vesical en etapas tempranas ${ }^{27}$. Los autores sugieren que antineoplásicos fuera del espectro de multirresistencia de la LRP podrían ser más efectivos en los tumores vesicales de bajo grado y estadio.

- Sobreexpresión de las proteínas antiapoptóticas Bcl-2 y Bcl-xL: Estudios in vitro con líneas celulares han demostrado que la sobreexpresión de la proteína Bcl-2 y Bcl-xL confieren resistencia celular a los agentes quimioterápicos. La adición de un oligonucleótido antisentido que disminuye los niveles de la proteína $\mathrm{Bcl}-2^{28}$ o de la $\mathrm{Bcl}-\mathrm{Xl}^{29}$ revierten estas resistencias.

- Sobreexpresión de la glutation-S-transferasa (GST) y aumento del glutation reducido (GSH): La GST es una enzima celular que biotransforma la TIO conjugándola con GSH en monoglutationiltiotepa o diglutationil-tiotepa, moléculas que son expulsadas al exterior de la célula mediante una bomba ATP-dependiente. Tanto la sobreexpresión de la GST como el aumento del GSH intracelular (sintetizado por la gamma-glutamil-cisteín sintetasa) confieren a las células tumorales una mayor capacidad de detoxificación y por tanto de resistencia a la tiotepa ${ }^{30,31}$ cisplatino $^{20}$ y otros citostá$\operatorname{ticos}^{32,33}$.

- Sobreexpresión de la metalotioneína: Es una proteína intracelular que se une a la MMC con gran afinidad impidiendo su acción sobre el ADN. $\mathrm{Su}$ cuantificación inmunohistoquímica tanto citoplásmica como nuclear en los tumores vesicales que recurrieron después de RTU seguida de tratamiento profiláctico con MMC fue significativamente mayor que en los que no recurrieron. La sobreexpresión de la metalotioneína predijo la resistencia del tumor a la terapia intravesical con MMC independientemente del grado tumoral ${ }^{34}$.

- Disminución de la expresión de la topoisomerasa II- $\beta$ (gen TOP2A): Esta enzima nuclear cataliza la ruptura y la nueva unión de la doble hélice del ADN imprescindibles para los procesos de transcripción, replicación y reparación del ADN. La sensibilidad de las células a las drogas que actúan inhibiendo la topoisomerasa II- $\alpha$ (antraciclinas) es mayor cuanto más cantidad de enzima exista en la célula. En líneas de tumor vesical resistentes a ADR se ha observado entre otros mecanismos de resistencia la disminución de los niveles de ARN mensajero (ARNm) correspondien- te a la topoisomerasa $\mathrm{II}^{35,36}$. En un estudio se tomaron muestras de los tumores antes de su tratamiento con EPI intravesical y, en los casos que recurrieron, después. Los niveles de ARNm de la topoisomerasa II en los tumores resistentes a la EPI (recurrentes) fueron significativamente menores que antes del tratamiento ${ }^{37}$. Además de su relación con la quimiorresistencia se ha visto que los niveles intranucleares de topoisomerasa II- $\alpha$ detectados por inmunohistoquimia tienen un valor pronóstico independiente del grado y del estadio tumoral. Niveles altos indican una mayor probabilidad de recurrencia y una disminución de la supervivencia ${ }^{38}$. En otro estudio la amplificación y sobreexpresión del gen TOP2A, además de asociarse a la amplificación del oncogen HER$2 / \mathrm{NEU}$, se relacionó con una menor supervivencia en tumores vesicales infiltrantes ${ }^{39}$.

- Sobreexpresión de la prolina-dirigida proteínkinasa F: Se ha visto que su sobreexpresión detectada por inmunohistoquimia se relaciona con la quimiorresistencia, recurrencias y progresión de los tumores vesicales. La supresión de su sobreexpresión mediante un oligonucleótido antisentido consigue potenciar la quimiosensibilidad a varios agentes quimioterápicos ${ }^{20}$.

- Sobreexpresión de la proteína FLICE inhibitoria (cFLIP): Su sobreexpresión confiere resistencia a la apoptosis receptor-inducida en estudios in vitro en tumores vesicales ${ }^{40}$.

- Sobreexpresión del receptor del IGF-1: Su bloqueo mediante un oligonucleótido antisentido consiguió en una línea celular sensibilizarla a la acción de la MMC, indicando que su sobreexpresión es un mecanismo de resistencia celular ${ }^{41,42}$.

\section{RESISTENCIA MULTICELULAR}

Los mecanismos de resistencia "multicelular" aparecen en los cultivos de agregados celulares tridimensionales, y no en las mismas células cultivadas en monocapa o en una suspensión de células individuales ${ }^{43-45}$. Muy explícito al respecto es un estudio de Kugler et $\mathrm{al}^{46}$ en el que 6 líneas celulares fueron cultivadas como monocapa y como esferoides celulares, y más tarde expuestas a varios agentes quimioterápicos durante 48 horas. Para todos los fármacos testados se observó una mayor resistencia en los esferoides en comparación con las monocapas. En el caso de los tumo- 
res vesicales Erlichman et $\mathrm{al}^{47}$ compararon la sensibilidad a la ADR en una línea de tumor vesical cultivada como monocapa, como esferoides y como xenoinjerto murino. Las células más superficiales de los esferoides mostraban la misma sensibilidad que las monocapas, pero las más internas eran más resistentes. Lo mismo se observó in vivo en los xenoinjertos.

Existen dos tipos de mecanismos implicados en la resistencia multicelular, los dependientes del contacto intercelular y los dependientes de la estructura tridimensional. Los primeros están mediados por las moléculas de adhesión y se presentan en cultivos en monocapa y en cultivos tridimensionales pero no en las suspensiones celulares, mientras que los segundos sólo se presentan en los cultivos tridimensionales.

\section{MECANISMOS DEPENDIENTES DEL CONTACTO INTERCELULAR}

- Interacciones célula-célula mediante cadherinas y célula-estroma mediante integrinas: Estas interacciones inducen la sobreexpresión del Bcl2 , proteína antiapoptótica. Ya se ha hablado del papel de las integrinas como transductores de señales de supervivencia, y de la pérdida de la adhesión como mecanismo extracelular activador de muerte. Se ha visto que cuando las células pierden estas interacciones se induce la apoptosis, fenómeno que describieron Frisch et $\mathrm{al}^{48} \mathrm{y}$ denominaron anoikis (en griego "sin casa"). En los tejidos normales el fenómeno anoikis protege de la diseminación de células sueltas y de su implantación en localizaciones anómalas. En los tumores sólidos y los esferoides tumorales la inhibición de la apoptosis en las células que están en contacto con su microentorno es uno de los mecanismos de la resistencia multicelular. Este fenómeno queda demostrado en estudios en los que la resistencia multicelular se revierte mediante proteasas que producen la disrupción de las uniones intercelulares. El mismo efecto reversor de la resistencia multicelular ejercen los anticuerpos monoclonales dirigidos contra las moléculas de adhesión ${ }^{45}$.

- Diferente expresión génica en los agregados celulares: El contacto intercelular produce una modulación en la expresión de factores de crecimiento, receptores celulares, proteínas regulado- ras del ciclo celular o enzimas nucleares implicadas en la replicación o reparación del $\mathrm{ADN}^{45}$. Por ejemplo, la p2 $7^{\mathrm{KIP} 1}$ es un inhibidor de las kinasas ciclín-dependientes que provoca la acumulación de células en las fases $G_{0}$ y $G_{1}$ del ciclo celular. Las células cuando confluyen sobreexpresan este inhibidor y se hacen más resistentes a muchos agentes quimioterápicos que encuentran menos células susceptibles de su acción. Otro ejemplo del que ya se ha hablado es la topoisomerasa II, que se expresa en niveles muy bajos cuando las células entran en contacto entre sí y su índice de proliferación es bajo, mientras que aumenta mucho en las células no confluyentes que crecen y se dividen rápidamente. Por este motivo los quimioterápicos inhibidores de la topoisomerasa II encuentran mayor resistencia en los cultivos tridimensionales o en las monocapas frente a los cultivos de suspensiones celulares.

\section{MECANISMOS DEPENDIENTES DE LA ESTRUCTURA TRIDIMENSIONAL}

- Permeabilidad de los agregados celulares a los fármacos: Se sabe que la penetración de las drogas en el tejido tumoral puede ser limitada, tal como demuestra un estudio con una línea de tumor vesical cultivada en una matriz de colágeno en el que se vio el enlentecimiento en la penetración de varias drogas radiomarcadas ${ }^{49}$. Dependiendo de lo lipofílicos que sean los fármacos o de su peso molecular la difusión en los esferoides puede variar ${ }^{50}$. Esto sugiere que in vivo pueden quedar células alejadas de los vasos en las que no se alcance una concentración letal y que, por tanto, la penetración de los fármacos puede ser una causa importante de resistencia a la quimioterapia en tumores sólidos. La ADR se ha visto que en esferoides celulares penetra sólo en las capas más superficiales y necesita concentraciones muy elevadas para llegar al centro de los esferoides ${ }^{51}$.

- Gradientes de oxígeno, nutrientes y proliferación: Existen otros mecanismos dependientes del crecimiento tridimensional de las células en los esferoides que provocan gradientes de oxígeno, de nutrientes y de proliferación así como otras características similares a las de los tumores sólidos, que también son responsables de la mayor resistencia en cultivos tridimensionales. Se sabe 
por ejemplo que la cantidad de células en diferentes fases del ciclo celular varía según la profundidad del esferoide, siendo las más proliferativas y más sensibles las que se encuentran en la superficie, mientras que en las zonas internas están las células quiescentes $\left(G_{0}\right)$ que son más resistentes ${ }^{50}$. Cuando la quimioterapia produce la muerte de las células más externas, las células quiescentes más internas pasan a la superficie donde están mejor oxigenadas y regresan al ciclo celular reanudando la cinética de crecimiento celular del tumor. Este fenómeno, implicado en la resistencia multicelular, se conoce como regrowth resistance (resistencia por repoblación) y fue descrito por Durand et $\mathrm{al}^{52}$. Se sabe también que la hipoxia que existe en el centro de los esferoides puede inducir la expresión de proteínas relacionadas con la resistencia como la GST o la metalotioneína ${ }^{45}$.

Existe una interrelación entre la resistencia multicelular y la multirresistencia "clásica" mediada por la glicoproteína P-170 (gen mdr-1). En células cultivadas como esferoides la resistencia mediada por el mdr-1 es más marcada que en las mismas células cultivadas como monocapas y, a la inversa, la resistencia multicelular parece verse favorecida por la resistencia "clásica"53.

Algunos autores ${ }^{54-56}$ encuentran in vitro altas resistencias a las antraciclinas EPI y ADR en comparación con la MMC o la TIO. Probablemente se debe a que la EPI y la ADR se ven mucho más afectadas por la resistencia multicelular que la MMC o la TIO. Phillips et $\mathrm{l}^{57}$ llegan a la conclusión de que la ADR y la EPI son menos activas en cultivos celulares en fase de plateau (como los cultivos tridimensionales) que en cultivos que se encuentran en fase de crecimiento exponencial (suspensiones), la TIO es igualmente citotóxica en ambas poblaciones celulares, y la MMC es más activa frente a las células en fase de plateau. Para explicar este hecho, hay que tener en cuenta que las antraciclinas ejercen su acción principalmente mediante la inhibición de la topoisomerasa II. Ya se ha dicho que esta enzima pasa a expresarse en niveles muy bajos cuando las células entran en contacto entre sí, se reduce el índice proliferativo y queda una mayor fracción de células a fase de reposo $\left(G_{0}\right)$, mientras que aumenta mucho su expresión en cultivos no confluyentes que tienen menor proporción de células en reposo y mayor en división. La MMC o la TIO son por el contrario agentes alquilantes puros capaces de dañar el ADN en la misma medida en las células en $G_{0}$ y en las células en división.

Uno de los mecanismos por el que actúan los agentes intravesicales es el de la prevención de la implantación de células tumorales durante la RTU. En este caso la quimioterapia actúa sobre células sueltas y la resistencia multicelular reviste una menor importancia, siendo aquí igual de efectivos todos los agentes. Sin embargo, los fármacos intravesicales también pueden actuar sobre tumores microscópicos coexistentes en el momento de la RTU o sobre tumores resecados de forma incompleta. En estos casos ya no se trata de células sueltas sino de estructuras tridimensionales donde sí puede tener importancia la resistencia multicelular y, por tanto, es posible que la MMC o la TIO sean más efectivas que la ADR o la EPI.

Pero trasladado a la clínica, ¿es realmente más efectiva la MMC?. Aunque clásicamente se ha dicho que ninguno de los agentes farmacológicos utilizados en instilación endovesical es superior al resto, en muchos de los estudios los resultados son mejores para la MMC aunque sin alcanzarse la significación estadística ${ }^{2,58-60}$. Reciente, Huncharek et $\mathrm{al}^{61-63}$ concluyen en sus metaanálisis que probablemente la MMC es más activa que otras drogas utilizadas vía intravesical y que la ADR es menos efectiva que el resto. En otro metaanálisis en el que se compara MMC con BCG en tumores de riesgo intermedio $\mathrm{y}$ alto Shelley et $\mathrm{al}^{64}$ no encuentran diferencias significativas en cuanto a la progresión y la supervivencia. Ningún otro agente quimioterápico intravesical ha logrado estos resultados.

\section{APLICACIÓN PRÁCTICA}

Veamos a continuación algunos ejemplos de las implicaciones que para el tratamiento puede tener el conocimiento de las resistencias y sus mecanismos.

La utilización de fármacos que revierten las resistencias se denomina quimioterapia de modulación. Consiste en la administración de un 
fármaco no citotóxico para permitir la acción del agente quimioterápico ${ }^{65}$. En el caso de los tumores vesicales superficiales el agente más estudiado ha sido el verapamilo, un antagonista de los canales de calcio que inhibe la función de la glicoproteína P-170. Ya se ha hecho mención a la función de esta proteína como canal de membrana por el que se bombean al exterior las antraciclinas y otros quimioterápicos, haciendo a las células resistentes a estos fármacos. Estudios in vitro con líneas celulares de tumor vesical, han puesto de manifiesto que en las células quimiosensibles la EPI se acumula sobre todo en el núcleo celular y algo en el citoplasma, mientras que en las resistentes los niveles globales de fluorescencia son menores, y el acúmulo se produce predominantemente en el citoplasma celular. El verapamilo aplicado a las células resistentes bloquea la glicoproteína P-170, consiguiendo que el patrón de distribución de la EPI sea similar al de las células sensibles ${ }^{66,67}$ y potenciando su acción citotóxica ${ }^{68}$.

La glicoproteína P-170 también puede ser inhibida por las hormonas esteroideas ${ }^{69}$, la estramustina $^{70} \mathrm{y}$ algunos isoprenoides ${ }^{71,72}$. Existe además una segunda generación de inhibidores de la glicoproteína P-170 como el biricodar (VX-710) o el valspodar (PSC833) pero que han resultado bastante tóxi$\cos ^{73,74}$. Otros moduladores de tercera generación con menor toxicidad como el tariquidar (XR9576), zosuquidar (LY335979), laniquidar (R101933) y el ONT-093 (OC-144-093) se están ensayando actualmente en estudios fases I y II ${ }^{73,75}$.

Otra línea de investigación abierta en los tumores vesicales multirresistentes es la de la sensibilización de las células tumorales a la apoptosis inducida por los linfocitos $\mathrm{T}$ y las células natural killer. En líneas celulares y en cultivos primarios de tumor vesical multirresistente se determinó la citotoxicidad inducida por el anticuerpo monoclonal anti-Fas, capaz al igual que el ligando Fas de activar la apoptosis por la vía del receptor de muerte. Se vio que sólo se inducía la apoptosis cuando se conseguía sobreexpresar el antígeno Fas (receptor Fas) en la superficie de las células mediante la administración conjunta de ADR. Esta sensibilización a la acción citotóxica de los linfocitos por la vía del receptor Fas se obtuvo con dosis pequeñas de $\mathrm{ADR}$, sugiriendo la aplicación in vivo de quimio e inmunoterapia combinada para el tratamiento de tumores vesicales resistentes ${ }^{76}$. En otro estudio, los isotiocianatos también demostraron ser capaces de sensibilizar las células de tumor vesical a la apoptosis por la vía del receptor de muerte ${ }^{77}$.

Para revertir la resistencia multicelular dependiente de la penetración de los fármacos en la mucosa vesical se han desarrollado diversas técnicas. La administración electromotriz multiplica entre 4 y 7 veces su transporte a través de las membranas biológicas en comparación con el transporte pasivo y consigue aumentar significativamente la eficacia de la quimioterapia intravesi$\mathrm{cal}^{78,79}$. La hipertermia local aplicada mediante un dispositivo intrauretral emisor de microondas también mejora la penetración de las drogas y su eficacia clínica ${ }^{80,81}$. En tumores de alto grado y CIS, la hipertermia ha mostrado eficacia no sólo en la profilaxis de recurrencias y la progresión sino también en la terapia ablativa ${ }^{82}$ y en el tratamiento de segunda línea tras fracasos de $\mathrm{BCG}^{80}$.

Conocida la existencia de resistencias a agentes individuales o de multirresistencias, y sabiendo que estas son variables de unos tumores a otros aunque tengan la misma histología, algunos investigadores han desarrollado test de sensibilidad. Es lógico pensar que los resultados en términos de disminución de recurrencias o de retraso en su aparición pueden mejorar si la elección de la droga se hace en base a un test de quimiosensibilidad. En el caso de los tumores transicionales superficiales existen diversos tests en la literatura para las drogas utilizadas intravesicalmente ${ }^{54,83-86}$. Su limitación más importante es que están basados en cultivos primarios en monocapa o en suspensión celular, por lo que sólo son capaces de reproducir in vitro los mecanismos de resistencia unicelulares. Nosotros estamos trabajando en un test de sensibilidad basado en cultivos tridimensionales, capaces de reproducir no sólo las resistencias unicelulares sino también las multicelulares ${ }^{87}$.

\section{REFERENCIAS}

1. Debruyne FMJ, Witjes JA. Can intravesical chemotherapy prevent progression of superficial bladder cancer? In: Kurth $\mathrm{KH}$, Mickisch GH, Schroder FH, editors. Renal, bladder and prostate cancer. An update. New York: The Parthenon Publishing Group 1999;223-226.

2. Kamat AM, Lamm DL. Intravesical therapy for bladder cancer. Urology 2000;55(2):161-168. 
3. Kurth KH, Bouffioux C, Sylvester R, van der Meijden AP, Oosterlinck W, Brausi M. Treatment of superficial bladder tumors: achievements and needs. The EORTC Genitourinary Group. Eur Urol 2000;37(3):1-9.

4. Witjes JA, Mungan NA, Debruyne FM. Management of superficial bladder cancer with intravesical chemotherapy: an update. Urology 2000;56(1):19-21.

5. Van der Meijden APM, Böhle A, Oosterlinck W, Lobel B, E. Rintala E, Solsona E, et al. Guidelines on non-muscle invasive bladder cancer: European Association of Urology; 2001.

6. Huncharek M, Geschwind JF, Witherspoon B, McGarry R, Adcock D. Intravesical chemotherapy prophylaxis in primary superficial bladder cancer: a meta-analysis of 3.703 patients from 11 randomized trials. $J$ Clin Epidemiol 2000;53(7):676-680

7. Lamm DL, Riggs DR, Traynelis CL, Nseyo UO. Apparent failure of current intravesical chemotherapy prophylaxis to influence the long-term course of superficial transitional cell carcinoma of the bladder. Journal of Urology 1995; 153(5): 1444-1450.

8. Kurth K, Tunn U, Ay R, Schroder FH, Pavone-Macaluso M, Debruyne F, et al. Adjuvant chemotherapy for superficial transitional cell bladder carcinoma: long-term results of a European Organization for Research and Treatment of Cancer randomized trial comparing doxorubicin, ethoglucid and transurethral resection alone. J Urol 1997;158(2): 378-384.

9. Martínez-Piñeiro L, Martínez-Piñeiro JA. Tratamiento del carcinoma vesical superficial. Quimioterapia local e inmunoterapia. Tratamiento del carcinoma in situ. In: Resel L, Moreno J, editors. Tratado de oncología urológica. Madrid: Sanidad y Ediciones, S.L.;2003;549-572.

10. Ivanchuk SM, Rutka JT. The cell cycle: accelerators, brakes, and checkpoints. Neurosurgery 2004;54(3):692-699; discussion 699-700.

11. DeWolf WC, Gaston SM. The cell cycle and its relevance to the urologist. J Urol 2004;171(4):1674-1681.

12. Skeel RT. Fundamentos biológicos y farmacológicos de la quimioterapia para el tratamiento del cáncer. In: Skeel RT, editor. Quimioterapia del cáncer. $5^{\underline{a}}$ ed. Madrid: Marbán Libros, S.L. 2000;3-19.

13. Gupta S. Molecular steps of cell suicide: an insight into immune senescence. J Clin Immunol 2000;20(4):229-239.

14. Solé FJ, Chechile G, Algaba F, Villaviciencio H, Cordon C. Regulación del ciclo celular. Bases moleculares de la carcinogénesis. In: Biología molecular de los tumores urológicos. Madrid: Ene Ediciones; 1995;61-66.

15. Gupta S. Molecular steps of death receptor and mitochondrial pathways of apoptosis. Life Sci 2001a;69(2526):2957-2964.

16. Gupta S. Molecular steps of tumor necrosis factor receptor-mediated apoptosis. Curr Mol Med 2001b;1(3):317324.

17. Benson MC, Giella J, Whang IS, Buttyan R, Hensle TW, Karp F, et al. Flow cytometric determination of the multidrug resistant phenotype in transitional cell cancer of the bladder: implications and applications. Journal of Urology 1991;146(4):982-986; discussion 986-987.

18. Guo H, Lu G, Xiong X, Dong J, Liu S. Establishment of doxorubicin-resistant human bladder cancer cell line (BUI-87/ADMR) and its mechanism of multidrug resistance. Chin Med J (Engl) 1997;110(3):167-172.
19. Hayes MC, Birch BR, Cooper AJ, Primrose JN. Cellular resistance to mitomycin $\mathrm{C}$ is associated with overexpression of MDR-1 in a urothelial cancer cell line (MGH-U1). Bju International 2001;87(3):245-250.

20. Hour TC, Chen J, Huang CY, Guan JY, Lu SH, Hsieh CY, et al. Characterization of chemoresistance mechanisms in a series of cisplatin-resistant transitional carcinoma cell lines. Anticancer Res 2000;20(5A):3221-3225.

21. Serretta V, Pavone C, Allegro R, Vella M, Sanguedolce R, Porcasi R, et al. Correlation between GP-170 expression, prognosis, and chemoresistance of superficial bladder carcinoma. J Cancer Res Clin Oncol 2003;129(8):472-476.

22. Tada Y, Wada M, Kuroiwa K, Kinugawa N, Harada T, Nagayama J, et al. MDR1 gene overexpression and altered degree of methylation at the promoter region in bladder cancer during chemotherapeutic treatment. 2000;6(12):4618-4627.

23. Zhu YY, Kong CZ, Zeng Y, Pang GY, Yang CM, Sun ZX. [The role of cell adhesion, multidrug resistance and cell proliferation in short-term recurrent cases with T1G3 superficial bladder cancer]. Zhonghua Wai Ke Za Zhi 2003;41 (12):893-896.

24. Sanguedolce R, Calascibetta A, Porcasi R, Melloni D, Pavone C, Tomasino RM, et al. Does P-glycoprotein-170 expression predict for chemoresistance in transitional cell carcinoma of the bladder? Anticancer Res 2002;22(5): 2971-2976.

25. Naito S, Hasegawa S, Yokomizo A, Koga H, Kotoh S, Kuwano M, et al. Non-P-glycoprotein-mediated atypical multidrug resistance in a human bladder cancer cell line. Jpn J Cancer Res 1995;86(11):1112-1118.

26. Tada Y, Wada M, Migita T, Nagayama J, Hinoshita E, Mochida Y, et al. Increased expression of multidrug resistance-associated proteins in bladder cancer during clinical course and drug resistance to doxorubicin. International Journal of Cancer. Journal International Du Cancer 2002;98(4):630-635.

27. Zhu Y, Kong C, Zeng Y, Sun Z, Gao H. Expression of lung resistance-related protein in transitional cell carcinoma of bladder. Urology 2004a;63(4):694-698.

28. Bilim V, Kasahara T, Noboru H, Takahashi K, Tomita Y. Caspase involved synergistic cytotoxicity of bcl-2 antisense oligonucleotides and adriamycin on transitional cell cancer cells. Cancer Letters 2000;155(2):191-198.

29. Lebedeva I, Raffo A, Rando R, Ojwang J, Cossum P, Stein CA. Chemosensitization of bladder carcinoma cells by bcl-xL antisense oligonucleotides. J Urol 2001;166(2):461-469.

30. Cnubben NH, Rommens AJ, Oudshoorn MJ, Van Bladeren PJ. Glutathione-dependent biotransformation of the alkylating drug thiotepa and transport of its metabolite monoglutathionylthiotepa in human MCF-7 breast cancer cells. Cancer Research 1998;58(20):4616-4623.

31. Dirven HA, Dictus EL, Broeders NL, van Ommen B, van Bladeren PJ. The role of human glutathione S-transferase isoenzymes in the formation of glutathione conjugates of the alkylating cytostatic drug thiotepa. Cancer Research 1995;55(8):1701-1706.

32. Yang CR, Ou YC, Kuo JH, Kao YL, Chen CL, Yean SY, et al. Intracellular glutathione content of urothelial cancer in correlation to chemotherapy response. Cancer Letters 1997;119(2): 157-162.

33. Yang W, Zeng X, Chen C, Chen Z, Du G. Correlative expression of glutathione S-transferase-pi and multidrug resistance associated protein in bladder transitional cell carcinoma. J Tongji Med Univ 2000;20(4):311-314. 
34. Lynn NN, Howe MC, Hale RJ, Collins GN, O’Reilly PH. Over expression of metallothionein predicts resistance of transitional cell carcinoma of bladder to intravesical mitomycin therapy. J Urol 2003;169(2):721-723.

35. Hasegawa S, Abe T, Naito S, Kotoh S, Kumazawa J, Hipfner DR, et al. Expression of multidrug resistanceassociated protein (MRP), MDR1 and DNA topoisomerase II in human multidrug-resistant bladder cancer cell lines. $\mathrm{Br}$ J Cancer 1995;71(5):907-913.

36. Kim WJ, Kakehi Y, Yoshida O. Multifactorial involvement of multidrug resistance-associated [correction of resistance] protein, DNA topoisomerase II and glutathione/glutathione-S-transferase in nonP-glycoprotein-mediated multidrug resistance in human bladder cancer cells. Int $\mathrm{J}$ Urol 1997;4(6):583-590.

37. Davies SL, Popert R, Coptcoat M, Hickson ID, Masters JR. Response to epirubicin in patients with superficial bladder cancer and expression of the topoisomerase II alpha and beta genes. Int J Cancer 1996a;65(1):63-66.

38. Koren R, Kugel V, Dekel Y, Weissman Y, Livne PM, Gal R. Human DNA topoisomerase-IIalpha expression as a prognostic factor for transitional cell carcinoma of the urinary bladder. BJU Int 2003;91(6):489-492.

39. Simon R, Atefy R, Wagner U, Forster T, Fijan A, Bruderer $\mathrm{J}$, et al. HER-2 and TOP2A coamplification in urinary bladder cancer. Int $J$ Cancer 2003;107(5):764-772.

40. Jonsson G, Paulie S, Grandien A. High level of cFLIP correlates with resistance to death receptor-induced apoptosis in bladder carcinoma cells. Anticancer Res 2003;23 (2B): 1213-1218.

41. Sun HZ, Wu SF, Tu ZH. Blockage of IGF-1R signaling sensitizes urinary bladder cancer cells to mitomycin-mediated cytotoxicity. Cell Res 2001a;11(2):107-115.

42. Sun HZ, Wu SF, Tu ZH. Knockdown of IGF-IR by Antisense Oligodeoxynucleotide auguments the sensitivity of bladder cancer cells to mitomycin. Acta Pharmacol Sin 2001b;22 (9):841-846.

43. Kobayashi H, Man S, Graham CH, Kapitain SJ, Teicher BA, Kerbel RS. Acquired multicellular-mediated resistance to alkylating agents in cancer. Proceedings of the National Academy of Sciences of the United States of America 1993;90(8):3294-3298.

44. Teicher BA, Herman TS, Holden SA, Wang YY, Pfeffer MR, Crawford JW, et al. Tumor resistance to alkylating agents conferred by mechanisms operative only in vivo. Science 1990;247(4949 Pt 1):1457-1461.

45. Desoize B, Jardillier J. Multicellular resistance: a paradigm for clinical resistance? Crit Rev Oncol Hematol 2000; 36(2-3):193-207.

46. Kugler A, Haschemi R, Zoller G, Gross AJ, Kallerhoff M, Ringert RH. In vitro investigations of new therapeutic agents on bladder tumor cell lines. Urol Res 1997;25(4):247-250.

47. Erlichman C, Vidgen D. Cytotoxicity of adriamycin in MGH-U1 cells grown as monolayer cultures, spheroids, and xenografts in immune-deprived mice. Cancer Res 1984;44(11):5369-5375.

48. Frisch SM, Francis H. Disruption of epithelial cell-matrix interactions induces apoptosis. J Cell Biol 1994;124(4): 619-626.

49. Tannock IF, Lee CM, Tunggal JK, Cowan DS, Egorin MJ. Limited penetration of anticancer drugs through tumor tissue: a potential cause of resistance of solid tumors to chemotherapy. Clinical Cancer Research: An Official Journal of the American Association For Cancer Research 2002;8(3):878-884.
50. Carlsson J, Nederman T. Tumor spheroids as a model in studies of drug effects. In: Bjerkvig R, editor. Spheroid culture in cancer research. Boca Raton: CRC Press;1992; 199-216.

51. Kerr DJ, Wheldon TE, Hydns S, Kaye SB. Cytotoxic drug penetration studies in multicellular tumour spheroids. Xenobiotica 1988;18(6):641-648.

52. Durand RE, Vanderbyl SL. Tumor resistance to therapy: a genetic or kinetic problem? Cancer Commun 1989;1(5): 277-283.

53. Kolchinsky A, Roninson IB. Drug resistance conferred by MDR1 expression in spheroids formed by glioblastoma cell lines. Anticancer Res 1997;17(5A):3321-3327.

54. Turkeri LN, Akdas G, Ozyurek M, Akdas A. The role of in vitro chemosensitivity tests to predict the clinical efficacy of antineoplastic agents in genito-urinary tumors. Arch Esp Urol 1998;51(4):398-404.

55. Uchibayashi T, Egawa M, Hisazumi H, Hasegawa T, Tanaka $\mathrm{T}$, Sasaki T. In vitro chemosensitivity test for human genitourinary tumors using collagen gel matrix. European Urology 1993;24(2):267-270.

56. Hirano Y, Kageyama S, Ushiyama T, Suzuki K, Fujita K. Clinical usefulness of chemotherapy based on an in vitro chemosensitivity test in urothelial cancer patients. Anticancer Res 2001;21(6A):4061-4066.

57. Phillips RM, Clayton MR. Plateau-phase cultures: an experimental model for identifying drugs which are bioactivated within the microenvironment of solid tumours. $\mathrm{Br}$ J Cancer 1997;75(2):196-201.

58. Huland H, Kloppel G, Feddersen I, Otto U, Brachmann W, Hubmann $\mathrm{H}$, et al. Comparison of different schedules of cytostatic intravesical instillations in patients with superficial bladder carcinoma: final evaluation of a prospective multicenter study with 419 patients. J Urol 1990;144(1): 68-71; discussion 71-72.

59. Paulson DF. Treatment of superficial carcinoma of the bladder. World Journal of Urology 1986;4:52-59.

60. Lamm DL. Carcinoma in situ. Urol Clin North Am 1992; 19(3):499-508.

61. Huncharek M, Kupelnick B. Impact of intravesical chemotherapy versus BCG immunotherapy on recurrence of superficial transitional cell carcinoma of the bladder: metaanalytic reevaluation. Am J Clin Oncol 2003;26(4): 402-407.

62. Huncharek M, Kupelnick B. The influence of intravesical therapy on progression of superficial transitional cell carcinoma of the bladder: a metaanalytic comparison of chemotherapy versus bacilli Calmette-Guerin immunotherapy. Am J Clin Oncol 2004;27(5):522-528.

63. Huncharek M, McGarry R, Kupelnick B. Impact of intravesical chemotherapy on recurrence rate of recurrent superficial transitional cell carcinoma of the bladder: results of a meta-analysis. Anticancer Res 2001;21(1B):765-769.

64. Shelley MD, Wilt TJ, Court J, Coles B, Kynaston H, Mason MD. Intravesical bacillus Calmette-Guerin is superior to mitomycin $\mathrm{C}$ in reducing tumour recurrence in high-risk superficial bladder cancer: a meta-analysis of randomized trials. BJU Int 2004;93(4):485-490.

65. Resel L, De la Peña E, Moreno J, Blanco E. Aspectos básicos de la quimioterapia en los tumores urológicos. In: Resel L, Moreno J, editors. Tratado de oncología urológica. Madrid: Sanidad y Ediciones, S.L. 2003;163-178. 
66. Davies CL, Duffy PM, MacRobert AJ, Loizidou MC, Cooper AJ, Taylor I. Localization of anthracycline accumulation in sensitive and resistant urothelial tumor cell lines. Cancer Detect Prev 1996b;20(6):625-633.

67. Duffy PM, Hayes MC, Gatrell SK, Cooper A, Smart CJ. Determination and reversal of resistance to epirubicin intravesical chemotherapy. A confocal imaging study. British Journal of Urology 1996a;77(6):824-829.

68. Harris NM, Duffy PM, Crook TJ, Anderson WR, Sharpe P, Hayes MC, et al. Intravesical pH: a potentially important variable affecting efficacy and the further development of anthracycline chemotherapy for superficial bladder cancer. BJU Int 2002a;90(9):957-964.

69. Lewin J, Cooper A, Birch B. Progesterone: a novel adjunct to intravesical chemotherapy. BJU Int 2002;90(7):736741.

70. Jennings AM, Solomon LZ, Sharpe P, Hayes MC, Cooper AJ, Birch BR. Estramustine reversal of resistance to intravesical epirubicin chemotherapy. European Urology 1999;35(4):327-335.

71. Enokida H, Gotanda T, Oku S, Imazono Y, Kubo H, Hanada T, et al. Reversal of P-glycoprotein-mediated paclitaxel resistance by new synthetic isoprenoids in human bladder cancer cell line. Jpn J Cancer Res 2002;93(9): 1037-1046.

72. Nakagawa M, Enokida H, Gotanda T, Tachiwada $T$, Imazono Y, Kubo H, et al. Taxol resistance and its reversal by synthetic isoprenoids in human bladder cancer cell line. Aktuelle Urol 2003;34(4):250-252.

73. Ross DD. Modulation of drug resistance transporters as a strategy for treating myelodysplastic syndrome. Best Pract Res Clin Haematol 2004;17(4):641-651.

74. Greenberg PL, Lee SJ, Advani R, Tallman MS, Sikic BI, Letendre L, et al. Mitoxantrone, etoposide, and cytarabine with or without valspodar in patients with relapsed or refractory acute myeloid leukemia and high-risk myelodysplastic syndrome: a phase III trial (E2995). J Clin Oncol 2004;22(6): 1078-1086.

75. Thomas H, Coley HM. Overcoming multidrug resistance in cancer: an update on the clinical strategy of inhibiting pglycoprotein. Cancer Control 2003;10(2):159-165.

76. Mizutani Y, Okada Y, Yoshida O, Fukumoto M, Bonavida B. Doxorubicin sensitizes human bladder carcinoma cells to Fas-mediated cytotoxicity. Cancer 1997;79(6):1180-1189.

77. Pullar JM, Thomson SJ, King MJ, Turnbull CI, Midwinter RG, Hampton MB. The chemopreventive agent phenethyl isothiocyanate sensitizes cells to Fas-mediated apoptosis. Carcinogenesis 2004;25(5):765-772.

78. Colombo R, Brausi M, Da_Pozzo L, Salonia A, Montorsi F, Scattoni V, et al. Thermo-chemotherapy and electromotive drug administration of mitomycin $\mathrm{C}$ in superficial bladder cancer eradication. a pilot study on marker lesion. European Urology 2001;39(1):95-100.
79. Di Stasi SM, Giannantoni A, Stephen RL, Capelli G, Navarra P, Massoud R, et al. Intravesical electromotive mitomycin $\mathrm{C}$ versus passive transport mitomycin $\mathrm{C}$ for high risk superficial bladder cancer: a prospective randomized study. J Urol 2003;170(3):777-782.

80. Van der Heijden AG, Kiemeney LA, Gofrit ON, Nativ O, Sidi A, Leib $Z$, et al. Preliminary European results of local microwave hyperthermia and chemotherapy treatment in intermediate or high risk superficial transitional cell carcinoma of the bladder. Eur Urol 2004;46(1):65-71; discussion 71-72.

81. Colombo R, Da Pozzo LF, Salonia A, Rigatti P, Leib Z, Baniel J, et al. Multicentric study comparing intravesical chemotherapy alone and with local microwave hyperthermia for prophylaxis of recurrence of superficial transitional cell carcinoma. J Clin Oncol 2003;21(23):4270-4276.

82. Gofrit ON, Shapiro A, Pode D, Sidi A, Nativ O, Leib Z, et al. Combined local bladder hyperthermia and intravesical chemotherapy for the treatment of high-grade superficial bladder cancer. Urology 2004;63(3):466-471.

83. Weaver DJ, Barrett BA, Ross G, Adelstein EH. Transitional explant reduction assay-a new in vitro testing system for intravesical chemotherapy. The Journal of Urology 1986; 135(2):386-391.

84. Irisawa C, Aikawa K, Ogihara M, Hashimoto T, Yamaguchi O, Shiraiwa Y, et al. [Clinical results of prophylactic intravesical chemotherapy on superficial bladder cancer using the dye exclusion assay]. Nippon Hinyokika Gakkai Zasshi 1992;83(11):1828-1834.

85. Gontero P, Sargent JM, Hopster DJ, Lewandowic GM, Taylor CG, Elgie AW, et al. Ex vivo chemosensitivity to mitomycin $\mathrm{C}$ in bladder cancer and its relationship with $\mathrm{P}$ glycoprotein and apoptotic factors. Anticancer Res 2002; 22(6C):4073-4080.

86. Anton-Aparicio LM, Urmeneta J, Borras M, Balaguer R, Berian JM. In vitro clonal assay as a predictive test of sensitivity to cytostatics in carcinoma of the human bladder. Actas Urol Esp 1985;9(6):517-522.

87. Burgués JP, Gómez L, Pontones JL, Vera Donoso CD, Jiménez-Cruz JF, Ozonas M. A chemosensitivity test for superficial bladder cancer based on three-dimensional culture of tumour spheroids. In: XXth EAU Congress. Abstract number 880. Istanbul 2005.

Dr. J.P. Burgués Gasión

Servicio de Urología. Hospital Univ. Son Dureta

Andrea Doria, 55

07014 Palma de Mallorca (Baleares)

e-mail: jpburgues@pulso.com

(Trabajo recibido el 25 de abril de 2005) 